\title{
Inverse relationship between autophagy and CTSK is related to bovine embryo quality
}

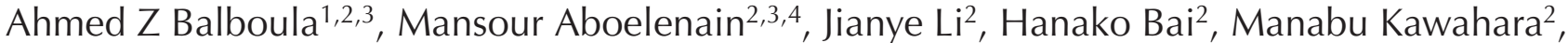 \\ Mohammed A Abdel-Ghani ${ }^{5}$ and Masashi Takahashi ${ }^{6,7}$ \\ ${ }^{1}$ Animal Sciences Research Center, University of Missouri, Columbia, Missouri, USA, ${ }^{2}$ Laboratory of Animal Breeding \\ and Reproduction, Graduate school of Agriculture, Hokkaido University, Hokkaido, Japan, ${ }^{3}$ Department of \\ Theriogenology, Faculty of Veterinary Medicine, Mansoura University, Mansoura, Egypt, ${ }^{4}$ Department of Genetics, \\ Rutgers University, Piscataway, New Jersey, USA, ${ }^{5}$ Department of Theriogenology, Faculty of Veterinary Medicine, \\ Assuit University, Assuit, Egypt, ${ }^{6}$ Research Faculty of Agriculture, Hokkaido University, Hokkaido, Japan and ${ }^{7}$ Global \\ Station for Food, Land and Water Resources, Global Institution for Collaborative Research and Education (GI-CoRE), \\ Hokkaido, Japan
}

Correspondence should be addressed to A Z Balboula or M Takahashi; Email: abalboula@missouri.edu ormmasashi@anim.agr.hokudai.ac.jp

\begin{abstract}
Improving the quality and the developmental competence of in vitro produced (IVP) embryos is an indispensable goal for assisted reproductive technology. Autophagy is a major protective mechanism for intracellular degradation of unnecessary cytoplasmic components. Autophagy ends by the fusion between autophagic vacuoles and lysosomes, allowing the degradation of the cargo by lysosomal enzymes, especially the cathepsins (CTSs). However, it is still unclear how autophagy and cathepsin K (CTSK) relate to embryo development. This study evaluated (1.) the activities of autophagy and CTSK in relation to bovine embryo quality and (2.) the effect of autophagy induction and/or CTSK inhibition on preimplantation embryo development and quality. We show here that good-quality embryos exhibited a greater autophagic activity and less CTSK activity compared to poor-quality embryos. Blastomeres of an individual embryo may vary in their quality. Good quality blastomeres showed an increased autophagic activity and decreased CTSK activity compared to poor-quality blastomeres within the same embryo at different developmental stages. Importantly, induction of autophagy and/or inhibition of CTSK improved the developmental rate (increased blastocyst and hatching rates) and the quality (increased total cell number and decreased the percentage of apoptotic cells) of IVP bovine embryos. These results demonstrate a promising approach to selectively isolate good-quality embryos and improve the efficiency of IVEP of cattle embryos. Reproduction (2020) 159 757-766
\end{abstract}

\section{Introduction}

In vitro embryo production (IVEP) is an efficient approach to produce and disseminate animals of superior genetic properties. Although IVEP technology has been greatly progressed in the recent years, the overall success remains lower than that in in vivo counterparts (AriasAlvarez et al. 2011, Han et al. 2017). Because reduced embryo quality is the major obstacle that compromises the success of IVEP and because morphological selection of embryos is less accurate and accompanied by poor outcomes (Deniz et al. 2018), it is essential to improve the efficiency of in vitro culture (IVC) conditions and identify biomarkers that allow evaluation and selection of high-quality embryos.

Autophagy is a major pathway for intracellular degradation of cytoplasmic components (Eskelinen 2005, Mizushima 2007, Agnello et al. 2015). The process starts with formation of an autophagosome that engulfs intra-cellular organelles. During the formation of autophagosomal membrane, cytoplasmic light chain 3B (LC3-I) binds to phosphatidylethanolamine to form the lipid modified conjugate (LC3-II). LC3-II is critical for autophagosome membrane expansion and fusion with the lysosome prior to the final degradation of the cargo by lysosomal enzymes (Mizushima 2007, Axe et al. 2008, Simonsen \& Tooze 2009, Weidberg et al. 2011). During stress, autophagy plays a pivotal role in maintaining cellular metabolism and homeostasis by selectively degrading unnecessary cytoplasmic components to provide nutrients and energy (Wu et al. 2012, Singh \& O'Reilly 2019). Autophagy is highly induced soon after fertilization and plays an important role during early embryonic development (Tsukamoto et al. 2008). Inhibition of autophagy during IVC impaired the developmental competence 
of preimplantation mouse embryos (Tsukamoto et al. 2008, Pan et al. 2020) and its induction improved the viability of cloned mouse embryos (Shen et al. 2015). Moreover, induction of autophagy using rapamycin during IVM or IVC improved the developmental competence of parthenogenetically produced porcine embryos (Lee et al. 2015, Elahi et al. 2017) as well as promoted the preattachment development of bovine embryos (Song et al. 2012), indicating that regulating autophagic activity is a promising approach to improve the efficiency of IVEP. Whether autophagic activity directly correlates with the developmental capacity of bovine embryos is still unknown.

The final step of autophagy-mediated intracellular degradation is the fusion between autophagosomes and lysosomes to form autolysosomes, necessary to degrade the cargo by lysosomal enzymes, especially the cathepsins (CTSs) (Galluzzi et al. 2014, Liu et al. 2017). CTSs constitute a major family of lysosomal proteases which play a key role in intracellular protein turnover. (Fonovic \& Turk 2014). Increased CTSs activity induces lysosomal membrane damage, and their leakage into the cytoplasm results in initiating caspase3/apoptotic cascade in different cell types pathway (Krippner et al. 1996, Adachi et al. 1997, Bossy-Wetzel et al. 1998, Scaffidi et al. 1998, Vancompernolle et al. 1998, Balboula et al. 2013). We previously demonstrated that active cathepsin B (CTSB) is expressed in bovine cumulus cells, oocytes and embryos and that its activity is inversely correlated with the quality of bovine oocytes and embryos (Balboula et al. 2010a,b, 2013). Importantly, we and others showed that inhibition of CTSB activity during in vitro maturation (IVM) and IVC improved the developmental competence and quality of bovine oocytes and embryos, respectively (Bettegowda et al. 2008, Balboula et al. 2010a,b, Min et al. 2014, Aboelenain et al. 2017). Furthermore, inhibition of CTSB activity rescued the adverse effect of heat-shock during IVM, in vitro fertilization (IVF) or IVC on subsequent bovine embryo development (Balboula et al. 2013, Yamanaka et al. 2018). The compromising effect of CTSB on embryonic quality has also been confirmed in other species. For example, inhibition of CTSB improved the quality and developmental competence of porcine and ovine oocytes and embryos (Kim et al. 2015, Pezhman et al. 2017, Liang et al. 2018). Interestingly, transcript abundances of Ctsb and Ctsk were upregulated in cumulus cells surrounding low developmentally competent bovine oocytes when compared to those surrounding high developmentally competent ones (Bettegowda et al. 2008). CTSK (also known as cathepsin O2) is predominantly expressed in osteoclast cells and ovaries (Bromme \& Okamoto 1995, Vizovisek et al. 2019) and its inhibition is used to treat osteoporosis in humans (Boggild et al. 2015). Although the involvement of CTSB in regulating oocyte and embryo quality has been established, data are lacking about the role of CTSK in regulating the developmental competence of preimplantation mammalian embryos and whether it correlates with embryonic quality.

Here, we find that CTSK and autophagic activities exhibit opposite patterns during early embryonic development. CTSK activity was increased in poorquality embryos, whereas autophagic activity was decreased in good-quality ones. Embryonic blastomeres vary in their quality. Inside the same embryo, we found that poor-quality cells showed high CTSK activity, while good-quality cells showed high autophagic activity. We then asked whether inhibition of CTSK activity and/or autophagy induction improves embryo developmental competence and quality. Supplementation of the IVC medium with rapamycin (Rap), an autophagy inducer, and/or odanactib (OD; a cell permeable CTSK inhibitor) improved the developmental competence and quality of bovine embryos. This study is the first to demonstrate the inverse relationship between CTSK and autophagic activities in relation to embryo quality and to provide evidence that manipulating CTSK/autophagy ratio is a promising approach to improve the developmental competence and quality of preimplantation mammalian embryos.

\section{Materials and methods}

Unless otherwise specified, all the chemicals and reagents used in this study were purchased from the MilliporeSigma, MO, USA.

\section{Oocytes collection and in vitro maturation (IVM)}

Holstein cow ovaries were collected from a local abattoir (Hayakita branch, Abira, Hokkaido). Aspiration of cumulus oocyte complexes (COCs) from medium-sized follicles (2-8 mm) was performed using an 18-gauge needle followed by washing three times in the maturation medium, Tissue Culture Medium (TCM) -199 (Invitrogen) supplemented with 5\% (V/V) fetal calf serum and follicle stimulating hormone (0.02 units $/ \mathrm{mL})$. COCs were then matured in a TCM-199 (50 COCs/500- $\mu \mathrm{L}$ drop) under mineral oil for $22 \mathrm{~h}$ at $38.5^{\circ} \mathrm{C}$ in a humidified atmosphere of $5 \% \mathrm{CO}_{2}$ in air.

\section{In vitro fertilization (IVF) and in vitro Culture (IVC)}

Straws of Holstein bull semen (from two fertile bulls) were thawed in a water bath at $37^{\circ} \mathrm{C}$ for $30 \mathrm{~s}$. Separation and washing of the actively motile sperm were done by centrifugation at $1800 \mathrm{~g}$ for $7 \mathrm{~min}$ in Brackett and Oliphant (BO) medium (Brackett \& Oliphant 1975) containing $2.5 \mathrm{mM}$ theophylline (Wako Pure Chemical Industries) and $7.5 \mu \mathrm{g} / \mathrm{mL}$ heparin sodium salt (Nacalai Tesque, Inv., Kyoto, Japan). After centrifugation, the sperm pellet was diluted with $\mathrm{BO}$ medium to get a final concentration of $5 \times 10^{6}$ spermatozoa per $\mathrm{mL}$. COCs were then incubated and fertilized by active motile sperm in droplets of modified BO's isotonic medium $(10 \mathrm{COCs} / 100 \mu \mathrm{L}$ ) containing $6 \mathrm{mg} / \mathrm{mL}$ fatty acid-free BSA and 
$2.5 \mathrm{mM}$ theophylline for $18 \mathrm{~h}$ at $38.5^{\circ} \mathrm{C}$ under a humidified atmosphere of $5 \% \mathrm{CO}_{2}$ in air. After fertilization, putative zygotes were denuded mechanically by repeated pipetting (Aono et al. 2013) followed by culturing in $50 \mu \mathrm{L}$ drops (20 putative zygotes/drop) of modified synthetic oviduct fluid medium supplemented with amino acid solution, $10 \mu / \mathrm{mL}$ insulin, $1 \mathrm{mM}$ glucose, and $3 \mathrm{mg} / \mathrm{mL}$ fatty acid-free BSA at $38.5^{\circ} \mathrm{C}$ under $5 \% \mathrm{CO}_{2}, 5 \% \mathrm{O}_{2}$ and $90 \% \mathrm{~N}_{2}$.

\section{Detection of intracellular CTSK activity}

Intracellular CTSK activity was detected by using a Magic red CTSK assay kit (Immunochemistry Technologies LLC, Bloomington, $M N$, USA) following the manufacturer instructions with some modifications. Briefly, embryos were incubated with the reaction mix $(2 \mu \mathrm{L}$ of DMSO-diluted stock solution in $500 \mu \mathrm{L}$ culture medium) for $25 \mathrm{~min}$ in a humidified atmosphere of $5 \% \mathrm{CO}_{2}$ at $38.5^{\circ} \mathrm{C}$. Nuclei staining was carried out by adding Hoechst $33342(25 \mu \mathrm{g} / \mathrm{mL})$ to the same staining solution for an additional 5 min. Embryos were then rinsed in PBS containing $0.5 \mathrm{mg} / \mathrm{mL}$ polyvinylpyrrolidone (PVP-PBS; Nacalai Tesque) prior to mounting onto a glass slide and observed under a fluorescence microscope (Leica Microsystems, Wetzlar, Germany). Nuclei were observed following excitation at $375 \mathrm{~nm}$ and intracellular CTSK activity was detected at $585 \mathrm{~nm}$ excitation. Images of CTSK activity within the same experiment were captured under the same parameters. Images were analyzed and the intensity of fluorescence was quantified using image J software (National Institutes of Health, Bethesda, MD, USA), keeping the processing parameters identical in all groups.

\section{Detection of autophagy activity}

Autophagic activity was detected using Cyto-ID Autophagy Detection kit (Enzo Life Sciences, Farmingdale, NY, USA) following the manufacturer's instructions with some modifications. Briefly, embryos were incubated with the reaction mix $\left(1 \mu \mathrm{L}\right.$ in $500 \mu \mathrm{L}$ culture medium) at $38.5^{\circ} \mathrm{C}$ for $25 \mathrm{~min}$. DNA was stained by adding Hoechst $33342(25 \mu \mathrm{g} / \mathrm{mL})$ to the same staining solution for an additional $5 \mathrm{~min}$. Embryos were then rinsed in PVP-PBS, mounted onto a glass slide, and imaged by fluorescence microscope (Leica) using $375 \mathrm{~nm}$ and $495 \mathrm{~nm}$ excitation filters to observe DNA and autophagy activity, respectively. The images were analyzed using the Image J software.

\section{Terminal deoxynucleotidyl transferase biotin-dUTP nick end labeling (TUNEL)}

Day 7 blastocysts were assessed for apoptosis using TUNEL assay kit (in situ cell death detection kit; Roche Applied Science). Blastocysts were fixed in $4 \%(\mathrm{w} / \mathrm{v})$ paraformaldehyde solution $(\mathrm{pH}$ 7.4) for $40 \mathrm{~min}$, rinsed three times in PVPPBS, and then permeabilized in PVP-PBS containing $0.5 \%$ TritonX-100 for $20 \mathrm{~min}$. After washing three times in PVPPBS for $5 \mathrm{~min}$ each, fragmented DNA ends of the cells were labeled with fluorescein-dUTP for $60 \mathrm{~min}$ at $38.5^{\circ} \mathrm{C}$. After incubation, blastocysts were washed three times in PVP-PBS for 5 min each, followed by mounting onto glass slides using mounting solution containing DAPI (Vectashield with DAPI; Vector Laboratories, Burlingame, CA, USA). Pretreatment of blastocysts with DNase I recombinant served as a positive control, while omitting fluorescein-dUTP served as a negative control. The fluorescence of the fragmented DNA ends was detected by a fluorescence microscope (Leica) using $495 \mathrm{~nm}$ excitation filter. The percentage of apoptotic positive cells was calculated for each embryo as follows: number of TUNELpositive nuclei/total number of nuclei in blastocyst $\times 100$.

\section{Statistical analysis}

One-way ANOVA and Student $t$-test were used to evaluate the differences between groups using GraphPad Prism. Oneway ANOVA was used to compare cleavage, blastocyst, and hatching rates among treated groups. ANOVA test was followed by the Tukey post hoc test. All percentage data were arcsine transformed prior to statistical analysis. The differences of $P<0.05$ were considered significant. Each experiment was replicated at least three times. The data were expressed as means \pm S.E.M.

\section{Experimental design}

Evaluation of CTSK and autophagic activities in relation to the developmental competence and quality of

bovine embryos

After IVF, bovine embryos were cultured for 5 days. Day 2 and 5 embryos were divided into two groups ('good' and 'poor' quality embryos) according to their developmental kinetics and morphology. In brief, embryos with normal morphology (symmetrical without any fragmentation) and fast development (have the developmental stage corresponding to each time point) (Lindner \& Wright 1983) were classified as good-quality embryos. On the other hand, slow-developing embryos that are lagging one cell cycle behind each time point with or without poor morphology (asymmetry and fragmentation) were classified as poor-quality embryos (Dinnyes et al. 1999, Lonergan et al. 1999, Yoshioka et al. 2000, Lequarre et al. 2003, Vandaele et al. 2007). To evaluate whether CTSK and autophagic activities are related to the quality of bovine embryos, good and poor-quality embryos were co-stained with Magic red CTSK assay kit and/or Cyto-ID Autophagy detection kit. Stained embryos were imaged by a fluorescence microscope at $495 \mathrm{~nm}$ and $585 \mathrm{~nm}$ excitation to observe autophagy and CTSK activity, respectively.

\section{Effect of autophagy induction and/or CTSK inhibition on the developmental competence and the quality of bovine preimplantation embryos}

To evaluate the effect of autophagy induction on the developmental competence of preimplantation embryos, putative zygotes (day 0) were cultured with a natural inducer of autophagic activity (Rap) at different concentrations (10, 50, or $100 \mathrm{nM})$. To evaluate the effect of CTSK inhibition, different concentrations $(1,5$, and $10 \mu \mathrm{M})$ of OD (MK-0822, 
Selleckchem, USA) were used. Both drugs (Rap or OD) were dissolved in DMSO and added to the IVC medium at day 0 for 7 days. DMSO-treated oocytes were employed as controls. The efficiency of Rap to stimulate autophagic activity and OD to inhibit CTSK was assessed in day 1 embryos using Cyto-ID Autophagy detection kit and magic red CTSK assay kit, respectively. The developmental competence was assessed by evaluating cleavage (day 2), blastocyst (day 7; including early and expanded), and hatching rates (day 7). The concentration that achieves the highest developmental rate of each treatment was selected for an additional experiment where the effect of combined autophagy induction and CTSK inhibition on developmental competence was evaluated. The quality for the produced blastocyst was assessed by evaluating the total cell number and the percentage of apoptotic cells in day 7 blastocysts.

\section{Results}

The quality of bovine embryo is associated with an inverse relationship between autophagy and

\section{CTSK activity}

To investigate the relationship between embryo quality and both CTSK and autophagic activities, days 2 or 5 embryos were classified into good and poor-quality embryos according to the criteria described previously prior to evaluating the activities of CTSK and autophagy using Magic-red CTSK Kit and Cyto-ID, respectively. We confirmed the specificity of both reagents in our system, where inhibiting CTSK using a cell permeable CTSK inhibitor (OD) reduced CTSK activity (Supplementary Fig. $1 \mathrm{~A}$ and $\mathrm{B}$, see section on supplementary materials given at the end of this article) and autophagy induction using Rap increased Cyto-ID autophagy signals (Supplementary Fig. 1C and D). Importantly, at each time point, autophagy exhibited an opposite pattern, where autophagy activity was greater in good-quality embryos when compared to poor-quality embryos (Fig. 1A, B, E and F). On the other hand, CTSK activity was greater in poor-quality embryos when compared to good-quality ones (Fig. 1A, C, E and G). Accordingly, autophagy/ CTSK ratio was greater in good-quality embryos than poor-quality embryos (Fig. 1D and $\mathrm{H}$ ).

\section{Inverse relationship between autophagy and CTSK activity is represented within blastomeres of the same embryo}

After fertilization, the 1-cell embryo divides mitotically. The produced cells from this cleavage are called blastomeres. During the previous experiment, we noticed that some embryos show a heterogenicity of autophagy and CTSK activity among blastomeres. The quality of embryonic blastomeres differ within the same embryo. To investigate whether the inverse relationship between CTSK and autophagic activities is related to blastomere quality, bovine embryos at

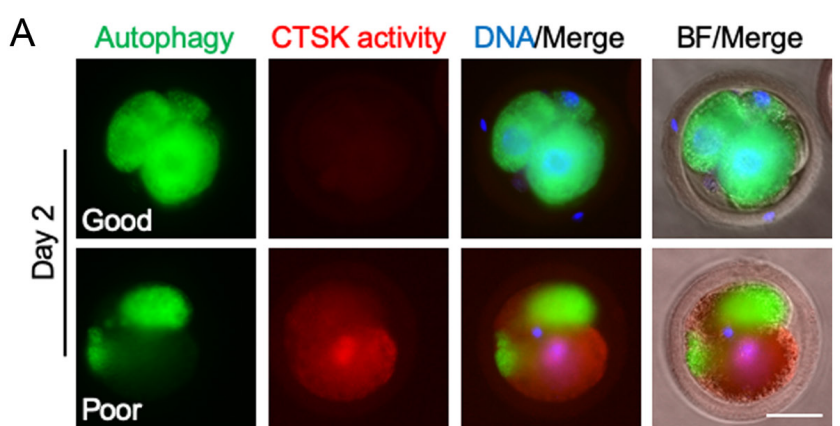

B
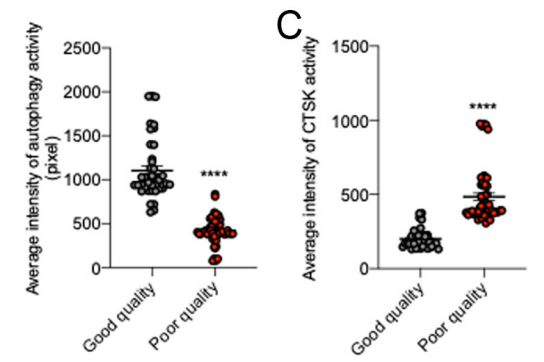

D

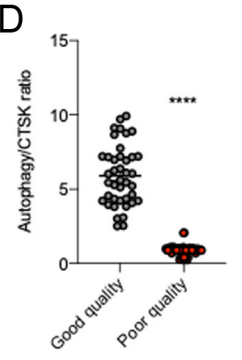

E

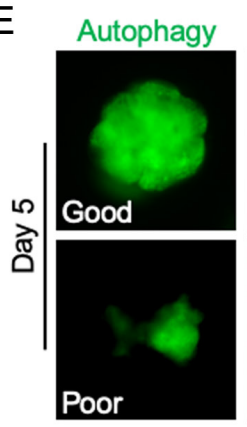

CTSK activity DNA/Merge

BF/Merge
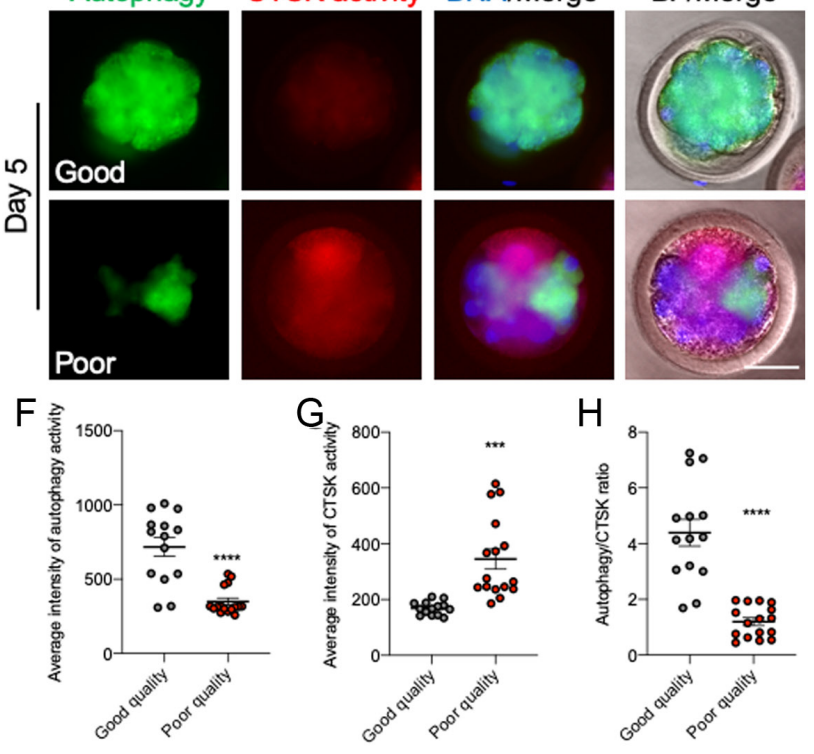

Figure 1 The quality of bovine embryos associates with inverse relationship between autophagy and CTSK activity. Day 2 and 5 bovine embryos were divided according to their quality into good and poor-quality embryos. Bovine embryos were co-stained with Cyto-ID Autophagy detection kit and Magic red CTSK assay kit to detect autophagy activity (green) and CTSK activity (red), respectively. DNA was detected via Hoechst staining (blue). Bright field (BF) images are shown in merge. Shown are representative images (scale bar, $100 \mu \mathrm{m}$ ) for good (upper row) and poor-quality (lower row) embryos at day 2 (A) and day 5 (E) after IVF. Average intensity of autophagy activity, CTSK activity as well as autophagy/CTSK ratio were quantified in day $2(\mathrm{~B}, \mathrm{C}$ and $\mathrm{D}$, respectively) and day 5 embryos ( $F, G$ and $H$, respectively). Experiments were repeated at least three times and total number of analyzed embryos is 154 . The data are expressed as mean \pm S.E.M. and Student $t$-test was used to analyze the data. Values with asterisks vary significantly, $* * * P<0.001, * * * * P<0.0001$. 
different stages (2-cell, 4 to 8-cell, and morula) of embryonic development were co-stained with Cyto-ID and Magic-red CTSK Kit to assess autophagy and CTSK activity, respectively. Interestingly, within each developmental stage, we found that morphologically normal blastomeres (light homogenous cytoplasm, regular plasma membrane borders, and without signs of vesiculation or fragmentation) (Hardarson et al. 2001) had a high level of autophagy and a low level of CTSK activity when compared to morphologically abnormal blastomeres in 2-cell (Fig. 2A), 4 to 8 cell (Fig. 2B), and morula (Fig. 2C) embryos.
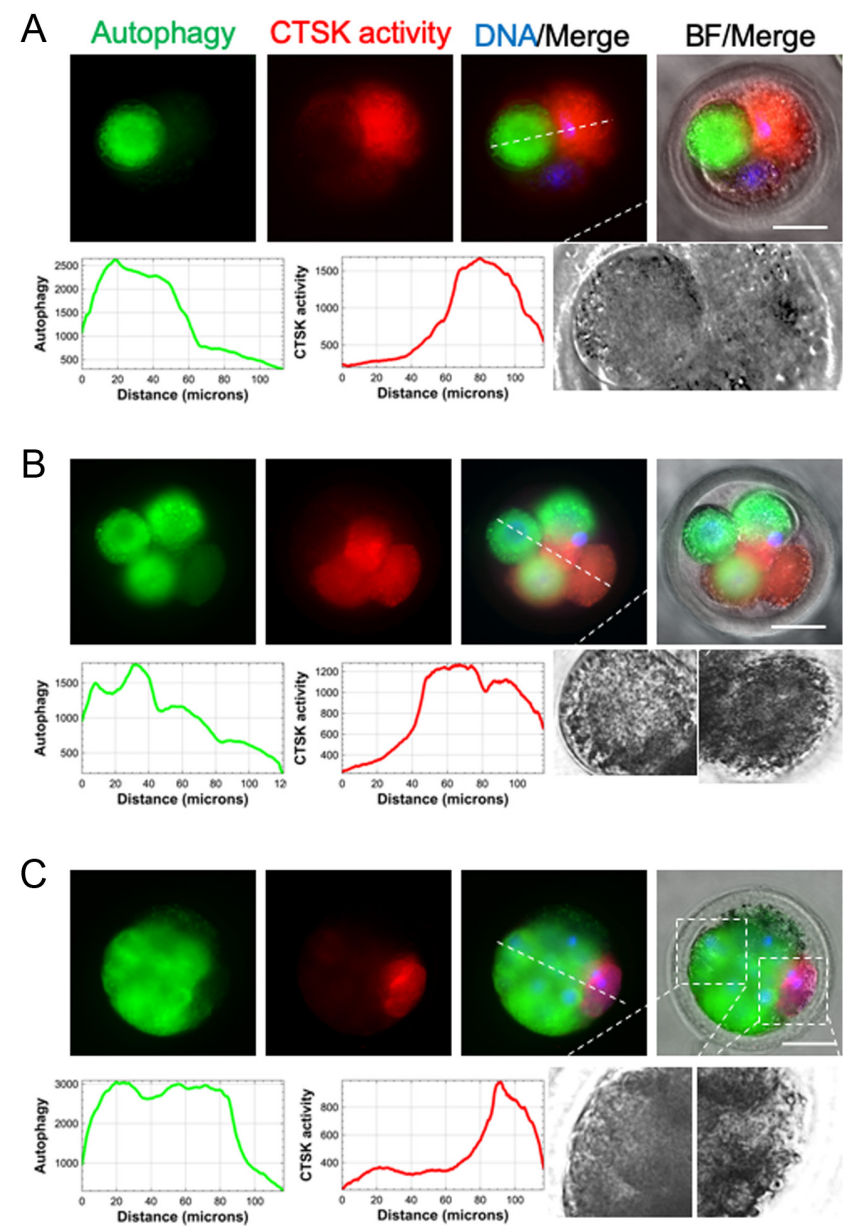

Figure 2 The quality of blastomeres of the individual embryo follows an inverse relationship between autophagy and CTSK activity. Bovine embryos at different stages (2-cell (A), 4-8-cell (B), and morula (C)) of embryonic development were co-stained with Cyto-ID Autophagy detection kit and Magic red CTSK assay kit to detect autophagy activity (green) and CTSK activity (red), respectively, in individual blastomeres. DNA was detected via Hoechst staining (blue). Bright field (BF) images are shown in merge. Poor-quality blastomeres (irregular and vesiculated) showed higher CTSK activity and lower autophagy than good-quality blastomeres. Lower panels: Examples of quantitative assessment of autophagy (green) and CTSK activities (red) using the 'plot profiles' function in ImageJ. Shown are representative images (scale bar, $100 \mu \mathrm{m}$ ). Experiments were repeated at least three times and total number of analyzed embryos is 154 .

\section{Inhibition of CTSK activity and/or induction of autophagy activity during IVC improves subsequent embryonic development}

Given the inverse relationship between CTSK activity and embryo quality, we hypothesized that the inhibition of CTSK during IVC might promote the subsequent embryonic development. To this end, different concentrations of a highly selective CTSK inhibitor, OD (Hao et al. 2015a,b, Bahuguna et al. 2016, Li et al. 2019), was added to the IVC medium and the developmental rates of bovine embryos were examined. Treatment of bovine embryos with OD during IVC did not affect the cleavage rate (Fig. 3A). However, the embryos cultured in the presence of $5 \mu \mathrm{M}$ OD displayed an increase in the rate of embryonic development to the blastocyst stage (Fig. 3B). Importantly, hatching rates were increased when embryos were cultured in a medium supplemented with $5 \mu \mathrm{M}$ OD (Fig. 3C).

Culturing bovine embryos with Rap during IVC had no effect on the cleavage rate (Fig. 4A). However, the embryos cultured in the presence of 50 or $100 \mathrm{nM}$ Rap showed an increase in the blastocyst rate (Fig. 4B). Hatching rates were increased when embryos were
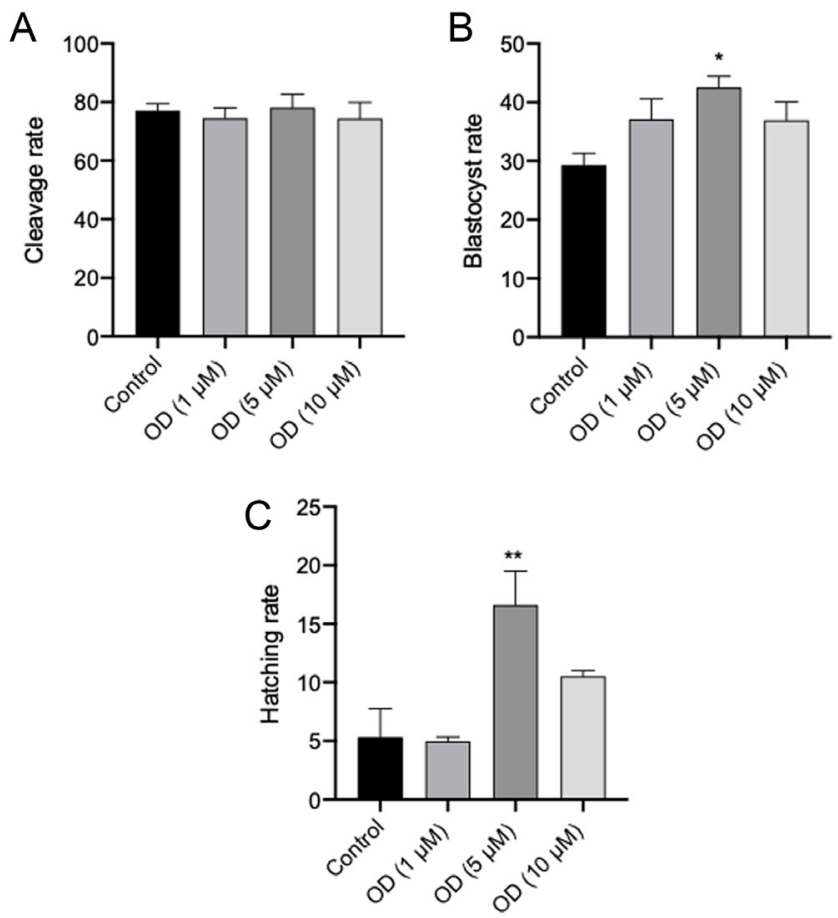

Figure 3 Effect of odanactib (OD) treatment during in vitro culture on subsequent cleavage, blastocyst, and hatching rates. After in vitro fertilization, putative zygotes were cultured for 7 days in culture medium supplemented without (DMSO control) or with 1, 5, or $10 \mu \mathrm{M}$ OD (CTSK inhibitor). Cleavage rate (A) was assessed on day 2, whereas blastocyst (B) and hatching rates (C) were observed on day 7. Experiments were repeated four times and total number of embryos is 333. The data are expressed as mean \pm S.E.M. and values with asterisks vary significantly, ${ }^{*} P<0.05,{ }^{* *} P<0.01$. 

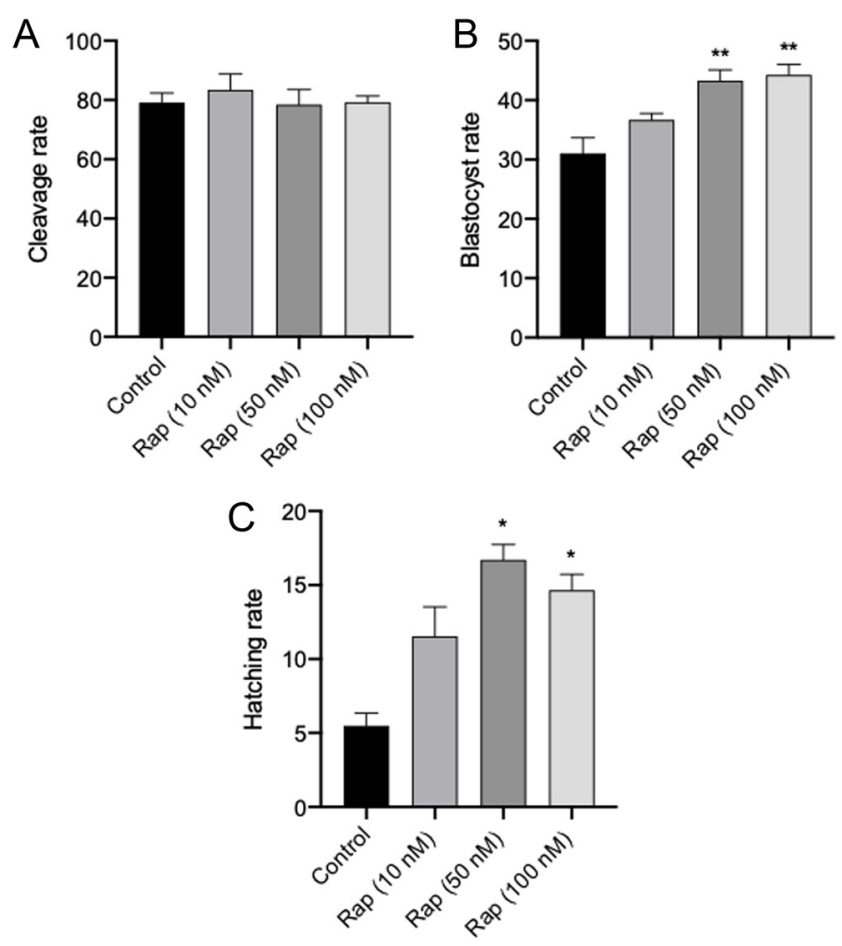

Figure 4 Effect of rapamycin (Rap) treatment during in vitro culture on subsequent cleavage, blastocyst, and hatching rates. After in vitro fertilization, putative zygotes were cultured for 7 days in culture medium supplemented without (DMSO control) or with 10, 50, or 100 nM Rap (autophagy inducer). Cleavage rate (A) was assessed on day 2, whereas blastocyst (B) and hatching rates $(C)$ were observed on day 7 . Experiments were repeated three times and total number of analyzed embryos is 274 . The data are expressed as mean \pm S.E.M. and values with asterisks vary significantly, ${ }^{*} P<0.05,{ }^{* *} P<0.01$.

cultured in a medium supplemented with 50 or $100 \mathrm{nM}$ Rap (Fig. 4C).

Because CTSK activity is inversely correlated with autophagic activity and because inhibition of CTSK activity or induction of autophagy improved the developmental rates of bovine embryos, we hypothesized that inhibition of CTSK and induction of autophagy simultaneously might have an additional improving effect. Strikingly, supplementation of the IVC medium with both $5 \mu \mathrm{M}$ OD and $50 \mathrm{nM}$ Rap (the lowest concentrations that achieved the highest developmental rates) increased the blastocyst and the hatching rates $(P<0.0001$, Fig. 5B and $\mathrm{C})$ but not the cleavage rates (Fig. 5A) when compared to control, OD, or Rap alone.

Average total cell number and the number of TUNEL apoptotic cells are considered valuable markers to judge embryo quality (Neuber et al. 2002, FouladiNashta et al. 2005). To investigate whether OD and/or Rap improves embryo quality, total cell number and the apoptotic status of day 7 blastocysts were assessed. The total cell number of blastocysts 7 days after addition of OD and/or Rap was increased than that in the control ones (Fig. 6A and B). The percentage of apoptotic cells
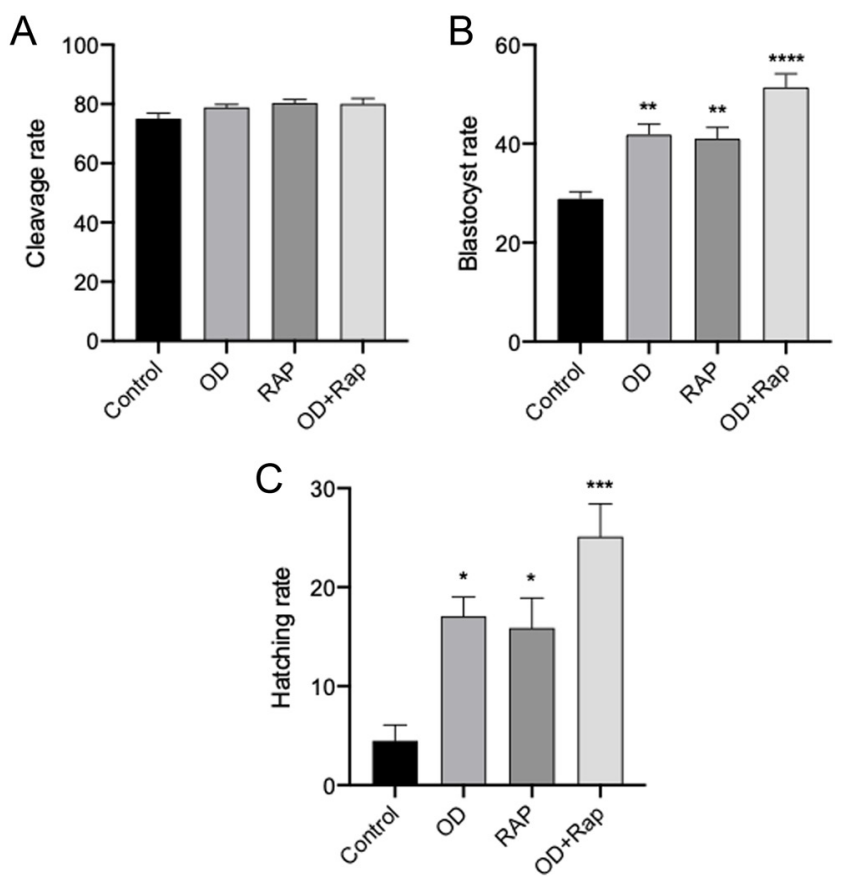

Figure 5 Rapamycin (Rap) and/or odanactib (OD) treatment during in vitro culture improved blastocyst and hatching rates of bovine embryos. After in vitro fertilization, putative zygotes were cultured for 7 days in culture medium supplemented with DMSO (control), OD $(5 \mu \mathrm{M})$, Rap $(50 \mu \mathrm{M})$, or OD + Rap. Cleavage rate (A) was assessed on day 2 , whereas blastocyst $(\mathrm{B})$ and hatching rates $(\mathrm{C})$ were observed on day 7 . Experiments were repeated five times and total number of analyzed embryos is 457 . The data are expressed as mean \pm S.E.M. and values with asterisks vary significantly, $* P<0.05,{ }^{* *} P<0.01$, $* * * P<0.001, * * * * P<0.0001$.

(TUNEL-positive cells) in the blastocysts derived from embryos cultured with OD and/or Rap was less than that in the control group (Fig. 6A and C). Taken together, these results strongly indicate that manipulating CTSK and autophagy levels is a promising approach to improve the developmental capacity and quality of IVP bovine embryos.

\section{Discussion}

In vitro production of embryos is an effective technology to improve genetic properties of animals and their production. However, major limitations of IVEP success are reduced developmental rate and reduced quality of the produced embryos. In this report, we found that CTSK activity is increased in poor-quality embryos when compared to good-quality ones. On the other hand, autophagy was increased in good-quality embryos than poor-quality embryos. Importantly, inhibition of CTSK activity and/or induction of autophagy during IVC improved the developmental competence and the quality of the produced embryos.

Autophagy is a process where cells degrade their unnecessary cellular components to provide energy and 
A
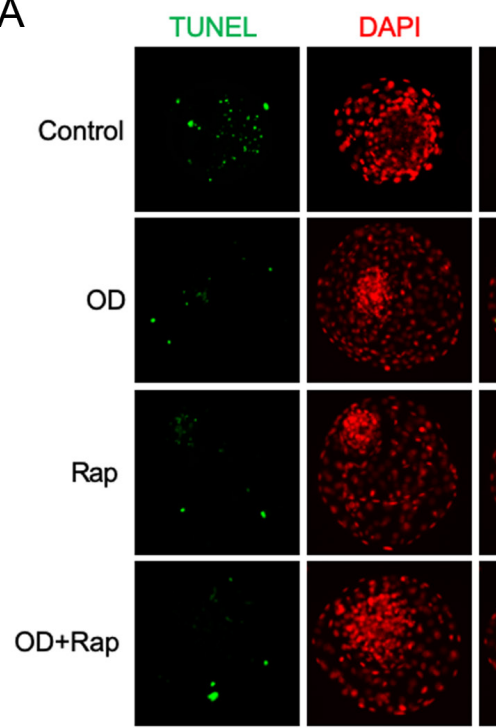

Merge

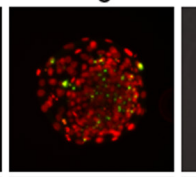

BF/Merge
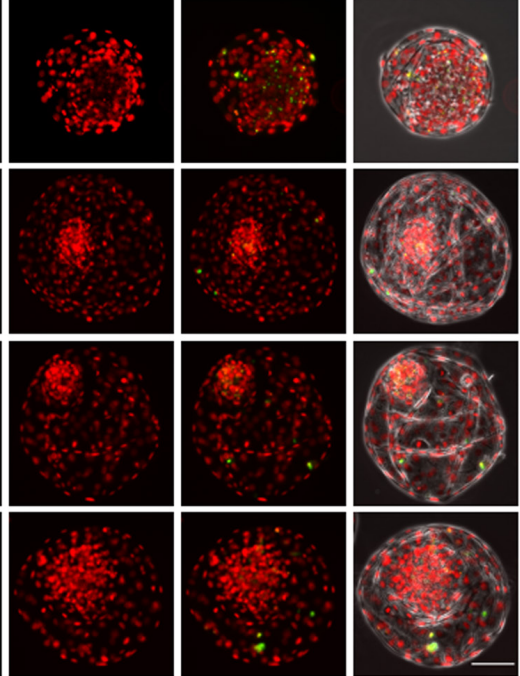

$\mathrm{B}$
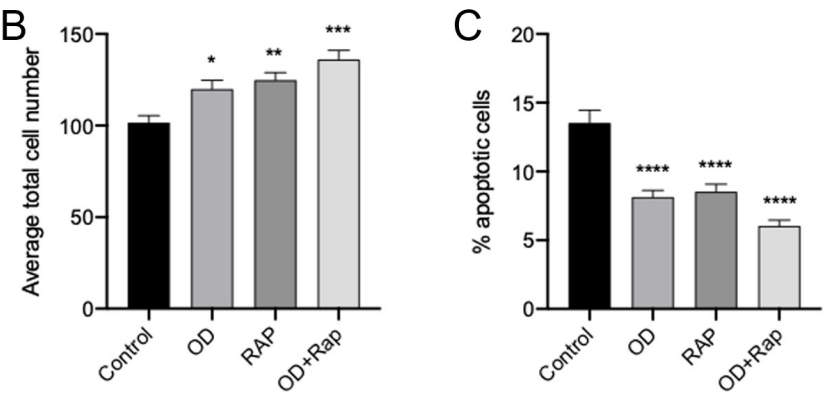

Figure 6 Effect of rapamycin (Rap) and/or odanactib (OD) treatment during in vitro culture on total cell number and apoptotic status of bovine blastocysts. After in vitro fertilization, putative zygotes were cultured for 7 days in culture medium supplemented with DMSO (control), OD (5 $\mathrm{MM}$ ), Rap (50 nM), or OD + Rap. (A) TUNEL staining (green) was used to detect apoptotic cells, while DAPI staining (red, pseudo color) was used for DNA detection and total cell number assessment. Shown are representative images (scale bar, $100 \mu \mathrm{m}$ ). (B) Quantification of average total cell number. (C) Quantification of TUNEL apoptotic positive cells. Experiments were replicated three times and total number of blastocysts examined is 84 . The data are expressed as mean \pm S.E.M. and values with asterisks vary significantly, $* P<0.05, * * P<0.01, * * * P<0.001, * * * * P<0.0001$.

nutrients (Eskelinen 2005, Mizushima 2007, Agnello et al. 2015). Autophagy is upregulated after fertilization in early mouse embryos (Tsukamoto et al. 2008). The relative expression of autophagy marker, LC3, was abundant in the zygote, reached the peak at the 2-cell and 4-cell stages, and gradually decreased from the morula to the blastocyst stage (Tsukamoto et al. 2008). We found a positive association between autophagy activity and bovine embryo quality, suggesting that autophagy activity is essential for early embryonic development. Indeed, the fertilization of mouse oocytes lacking Autophagy Related 5 (ATG5) with Atg5\% sperm resulted in a failure of embryonic development beyond 4-cell stage (Tsukamoto et al. 2008). Moreover, induction of autophagy using rapamycin and pp242 during somatic cell nuclear transfer (SCNT) enhanced the development of SCNT embryos to the blastocyst stage (Shen et al. 2015). In bovine embryos, autophagy is inversely correlated with endoplasmic reticulum stress (Song et al. 2012). This finding comes in consistence with our observations of high autophagy activity in good-quality embryos because early bovine embryo development requires appropriate balance between autophagy and endoplasmic reticulum stress (Song et al. 2012). The improving effect of autophagy induction using Rap during IVC further confirms the importance of autophagy during early development, likely through degrading unrequired maternal RNAs and proteins after maternal zygotic activation as well as providing nutrients and energy (Mizushima et al. 2004, Tsukamoto et al. 2008, Mizushima \& Levine 2010).

Although lysosomal cathepsins are ubiquitously expressed in different cell types, CTSK (cathepsin O2) showed predominant levels of expression specifically in osteoclast cells, cancer cells, and ovaries (Bromme \& Okamoto 1995, Vizovisek et al. 2019). The role of CTSK in the ovary has not been studied. Here, we showed that CTSK activity is expressed in early stage bovine embryos, albeit increased in poor-quality ones. Our previous studies demonstrated that CTSB activity, another member of lysosomal cysteine protease family, is inversely correlated with the quality of bovine embryos (Balboula et al. 2010b). Exposure of lysosomes to a moderate stress causes CTSB leakage into the cytoplasm (Brunk et al. 1997). However, increased intra-lysosomal CTSB activity can promote lysosomal membrane permeability leading ultimately to its leakage (Werneburg et al. 2002, Johansson et al. 2010). In both cases, leakage of CTSB into the cytoplasm is the key player for initiating caspase 3/apoptotic pathway (Krippner et al. 1996, Adachi et al. 1997, Bossy-Wetzel et al. 1998, Scaffidi et al. 1998, Vancompernolle et al. 1998). Similar to our CTSK results, inhibition of CTSB activity during IVC decreased the number of TUNEL-positive (apoptotic) cells and improved the developmental competence and quality of bovine embryos (Balboula et al. 2010b). Taken together, CTSK seems to have the same compromising effect as CTSB does on the quality of bovine embryos. OD selectively binds to and inhibits CTSK activity in different cell types (Hao et al. 2015a,b, Bahuguna et al. 2016, Li et al. 2019). To our knowledge, OD does not have the ability to inhibit other cathepsins or cysteine proteases (Gauthier et al. 2008). Owing to its ability to inhibit CTSK and its ability to reduce bone resorption while maintaining bone formation, OD was used to treat osteoporosis in humans (Boggild et al. 2015). Here, we found that CTSK inhibition by OD during IVC improved preimplantation embryonic development in bovine, supporting the notion that regulating lysosomal CTSs is a promising approach to improve the efficiency of IVEP.

Identifying markers for good-quality embryos has been an essential goal for efficient IVEP either in animals or 
humans. We found an opposite pattern between CTSK and autophagy in relation to embryo quality. Good-quality but not poor-quality embryos exhibited high autophagy and low CTSK activity. This result suggests that high CTSK activity could be used as a marker of poor-quality embryos, whereas high autophagy activity indicates good-quality embryos. Although each of CTSK and autophagy activities could be used a quality marker, the ratio of autophagy/CTSK within the same developmental stage is a more accurate predictor of embryo quality, as it excludes the possible effect of fluorescence bleaching and imaging variations among different experiments that require live cells. Determining the cut-off point of this ratio will be a helpful tool, as a quality marker to improve the efficiency of assisted reproductive technology.

The relationship between apoptosis and autophagy is still controversial. Although autophagy inhibits apoptosis in certain type of cells, it can also stimulate apoptosis in special cases (Marino et al. 2014). Although we have not addressed the underlying mechanism of this opposite pattern between lysosomal CTSK and autophagy, different possibilities could be considered. One possibility is that the activity of autophagy or CTSK negatively regulates the other. For example, suppression of lysosomal enzymes induces autophagy via a feedback down-regulation of MTOR Complex 1 (Li et al. 2013). On the other hand, autophagy has the ability to inhibit apoptosis by blocking the activation of cytoplasmic caspases (Thorburn 2008, Song et al. 2017). However, the additive improving effect of combined OD+Rap over OD or Rap alone suggests that autophagy and CTSK have non-overlapping pathways to determine the quality of early stage embryos. Further investigations are required to dissect these possibilities. In conclusion, the results reported here reveal that autophagy and CTSK activities could be used as markers of bovine embryo quality. Moreover, induction of autophagy and/or inhibition of CTSK during IVC are promising approaches to improve the developmental rate and quality of IVP bovine embryos.

\section{Supplementary materials}

This is linked to the online version of the paper at https://doi. org/10.1530/REP-20-0036.

\section{Declaration of interest}

The authors declare that there is no conflict of interest that could be perceived as prejudicing the impartiality of the research reported.

\section{Funding}

This study was supported by grant-aid for scientific research from JSPS (KAKENHI, 24580439 and 24780265) to M T and A Z B (P15400) and laboratory start-up funding from the University of Missouri to A Z B.

\section{Author contribution statement}

$A$ Z B and M T conceived the study. A Z B performed the experiments. A Z B, M A, J L, M A, H B, and $M K$ analyzed data and prepared the figures. A Z B and M T wrote the paper.

\section{Acknowledgements}

The authors would like to thank members of the laboratory of Animal Breeding and Reproduction, Hokkaido University, for discussions and support. The authors thank Dr Rocio Rivera, University of Missouri, for critical reading of the manuscript.

\section{References}

Aboelenain $M$, Balboula AZ, Kawahara M, El-Monem Montaser A, Zaabel SM, Kim SW, Nagano M \& Takahashi M 2017 Pyridoxine supplementation during oocyte maturation improves the development and quality of bovine preimplantation embryos. Theriogenology 91 127-133. (https://doi.org/10.1016/j.theriogenology.2016.12.022)

Adachi S, Cross AR, Babior BM \& Gottlieb RA 1997 Bcl-2 and the outer mitochondrial membrane in the inactivation of cytochrome c during Fasmediated apoptosis. Journal of Biological Chemistry 272 21878-21882. (https://doi.org/10.1074/jbc.272.35.21878)

Agnello M, Bosco L, Chiarelli R, Martino C \& Roccheri MC 2015 The Role of Autophagy and Apoptosis during Embryo Development.In Cell Death - Autophagy, Apoptosis and Necrosis Ed Tobias M Ntuli IntechOpen. (https://doi.org/10.5772/61765)

Aono A, Nagatomo H, Takuma T, Nonaka R, Ono Y, Wada Y, Abe Y, Takahashi M, Watanabe T \& Kawahara M 2013 Dynamics of intracellular phospholipid membrane organization during oocyte maturation and successful vitrification of immature oocytes retrieved by ovum pick-up in cattle. Theriogenology 79 1146.e1-1152.e1. (https://doi.org/10.1016/j. theriogenology.2013.02.009)

Arias-Alvarez M, Bermejo-Alvarez P, Gutierrez-Adan A, Rizos D, Lorenzo PL \& Lonergan P 2011 Effect of leptin supplementation during in vitro oocyte maturation and embryo culture on bovine embryo development and gene expression patterns. Theriogenology 75 887-896. (https://doi.org/10.1016/j.theriogenology.2010.10.031)

Axe EL, Walker SA, Manifava M, Chandra P, Roderick HL, Habermann A, Griffiths G \& Ktistakis NT 2008 Autophagosome formation from membrane compartments enriched in phosphatidylinositol 3-phosphate and dynamically connected to the endoplasmic reticulum. Journal of Cell Biology 182 685-701. (https://doi.org/10.1083/jcb.200803137)

Bahuguna R, Jain A, Khan SA \& Arvind MS 2016 Role of odanacatib in reducing bone loss due to endodontic disease: an overview. Journal of International Society of Preventive and Community Dentistry 6 S175S181. (https://doi.org/10.4103/2231-0762.197183)

Balboula AZ, Yamanaka K, Sakatani M, Hegab AO, Zaabel SM \& Takahashi M 2010a Cathepsin B activity is related to the quality of bovine cumulus oocyte complexes and its inhibition can improve their developmental competence. Molecular Reproduction and Development 77 439-448. (https://doi.org/10.1002/mrd.21164)

Balboula AZ, Yamanaka K, Sakatani M, Hegab AO, Zaabel SM \& Takahashi M 2010b Intracellular cathepsin B activity is inversely correlated with the quality and developmental competence of bovine preimplantation embryos. Molecular Reproduction and Development 77 1031-1039. (https://doi.org/10.1002/mrd.21250)

Balboula AZ, Yamanaka K, Sakatani M, Kawahara M, Hegab AO, Zaabel SM \& Takahashi M 2013 Cathepsin B activity has a crucial role in the developmental competence of bovine cumulus-oocyte complexes exposed to heat shock during in vitro maturation. Reproduction 146 407-417. (https://doi.org/10.1530/REP-13-0179)

Bettegowda A, Patel OV, Lee KB, Park KE, Salem M, Yao J, Ireland JJ \& Smith GW 2008 Identification of novel bovine cumulus cell molecular markers predictive of oocyte competence: functional and diagnostic implications. Biology of Reproduction 79 301-309. (https://doi. org/10.1095/biolreprod.107.067223) 
Boggild MK, Gajic-Veljanoski O, Mcdonald-Blumer H, Ridout R, Tile L, Josse R \& Cheung AM 2015 Odanacatib for the treatment of osteoporosis. Expert Opinion on Pharmacotherapy 16 1717-1726. (https://doi.org/10. 1517/14656566.2015.1064897)

Bossy-Wetzel E, Newmeyer DD \& Green DR 1998 Mitochondrial cytochrome $\mathrm{c}$ release in apoptosis occurs upstream of DEVD-specific caspase activation and independently of mitochondrial transmembrane depolarization. EMBO Journal 17 37-49. (https://doi.org/10.1093/ emboj/17.1.37)

Brackett BG \& Oliphant G 1975 Capacitation of rabbit spermatozoa in vitro. Biology of Reproduction 12 260-274. (https://doi.org/10.1095/ biolreprod12.2.260)

Bromme D \& Okamoto K 1995 Human cathepsin O2, a novel cysteine protease highly expressed in osteoclastomas and ovary molecular cloning, sequencing and tissue distribution. Biological Chemistry HoppeSeyler 376 379-384. (https://doi.org/10.1515/bchm3.1995.376.6.379)

Brunk UT, Dalen H, Roberg K \& Hellquist HB 1997 Photo-oxidative disruption of lysosomal membranes causes apoptosis of cultured human fibroblasts. Free Radical Biology and Medicine 23 616-626. (https://doi. org/10.1016/s0891-5849(97)00007-5)

Deniz FP, Encinas C \& Fuente J 2018 Morphological embryo selection: an elective single embryo transfer proposal. JBRA Assisted Reproduction 22 20-25. (https://doi.org/10.5935/1518-0557.20180015)

Dinnyes A, Lonergan P, Fair T, Boland MP \& Yang X 1999 Timing of the first cleavage post-insemination affects cryosurvival of in vitro-produced bovine blastocysts. Molecular Reproduction and Development $\mathbf{5 3}$ 318-324. 2795(199907)53:3<318::AID-MRD7>3.0.CO;2-O)

Elahi F, Lee H, Lee J, Lee ST, Park CK, Hyun SH \& Lee E 2017 Effect of rapamycin treatment during post-activation and/or in vitro culture on embryonic development after parthenogenesis and in vitro fertilization in pigs. Reproduction in Domestic Animals 52 741-748. (https://doi. org/10.1111/rda.12974)

Eskelinen EL 2005 Maturation of autophagic vacuoles in Mammalian cells. Autophagy 1 1-10. (https://doi.org/10.4161/auto.1.1.1270)

Farin PW, Slenning BD \& Britt JH 1999 Estimates of pregnancy outcomes based on selection of bovine embryos produced in vivo or in vitro. Theriogenology 52 659-670. (https://doi.org/10.1016/S0093691X(99)00160-0)

Fonovic M \& Turk B 2014 Cysteine cathepsins and extracellular matrix degradation. Biochimica and Biophysica Acta 1840 2560-2570. (https:// doi.org/10.1016/j.bbagen.2014.03.017)

Fouladi-Nashta AA, Alberio R, Kafi M, Nicholas B, Campbell KH \& Webb R 2005 Differential staining combined with TUNEL labelling to detect apoptosis in preimplantation bovine embryos. Reproductive Biomedicine Online 10 497-502. (https://doi.org/10.1016/s14726483(10)60827-9)

Galluzzi L, Pietrocola F, Levine B \& Kroemer G 2014 Metabolic control of autophagy. Cell 159 1263-1276. (https://doi.org/10.1016/j. cell.2014.11.006)

Gauthier JY, Chauret N, Cromlish W, Desmarais S, Duong LT, Falgueyret JP, Kimmel DB, Lamontagne S, Leger S, Leriche T et al. 2008 The discovery of odanacatib (MK-0822), a selective inhibitor of cathepsin K. Bioorganic and Medicinal Chemistry Letters 18 923-928. (https://doi.org/10.1016/j. bmcl.2007.12.047)

Han HI, Lee SH \& Park CK 2017 Development of in vitro embryo production system using collagen matrix gel attached with vascular endothelial growth factor derived from interleukin-1 beta-treated porcine endometrial tissue. Tissue Engineering: Part C, Methods 23 396-403. (https://doi.org/10.1089/ten.TEC.2017.0071)

Hao L, Chen J, Zhu Z, Reddy MS, Mountz JD, Chen W \& Li YP 2015a Odanacatib, A cathepsin K-specific inhibitor, inhibits inflammation and bone loss caused by periodontal diseases. Journal of Periodontology $\mathbf{8 6}$ 972-983. (https://doi.org/10.1902/jop.2015.140643)

Hao L, Chen W, Mcconnell M, Zhu Z, Li S, Reddy M, Eleazer PD, Wang M \& Li YP 2015b A small molecule, odanacatib, inhibits inflammation and bone loss caused by endodontic disease. Infection and Immunity $\mathbf{8 3}$ 1235-1245. (https://doi.org/10.1128/IAl.01713-14)

Hardarson T, Hanson C, Sjogren A \& Lundin K 2001 Human embryos with unevenly sized blastomeres have lower pregnancy and implantation rates: indications for aneuploidy and multinucleation. Human Reproduction 16 313-318. (https://doi.org/10.1093/humrep/16.2.313)
Johansson AC, Appelqvist H, Nilsson C, Kagedal K, Roberg K \& Ollinger K 2010 Regulation of apoptosis-associated lysosomal membrane permeabilization. Apoptosis 15 527-540. (https://doi.org/10.1007/ s10495-009-0452-5)

Kim SH, Zhao MH, Liang S, Cui XS \& Kim NH 2015 Inhibition of cathepsin $\mathrm{B}$ activity reduces apoptosis by preventing cytochrome $\mathrm{c}$ release from mitochondria in porcine parthenotes. Journal of Reproduction and Development 61 261-268. (https://doi.org/10.1262/jrd.2015-019)

Krippner A, Matsuno-Yagi A, Gottlieb RA \& Babior BM 1996 Loss of function of cytochrome $\mathrm{C}$ in Jurkat cells undergoing fas-mediated apoptosis. Journal of Biological Chemistry 271 21629-21636. (https:// doi.org/10.1074/jbc.271.35.21629)

Lee J, Park JI, Yun JI, Lee Y, Yong H, Lee ST, Park CK, Hyun SH, Lee GS \& Lee E 2015 Rapamycin treatment during in vitro maturation of oocytes improves embryonic development after parthenogenesis and somatic cell nuclear transfer in pigs. Journal of Veterinary Science 16 373-380. (https://doi.org/10.4142/jvs.2015.16.3.373)

Lequarre AS, Marchandise J, Moreau B, Massip A \& Donnay I 2003 Cell cycle duration at the time of maternal zygotic transition for in vitro produced bovine embryos: effect of oxygen tension and transcription inhibition. Biology of Reproduction 69 1707-1713. (https://doi. org/10.1095/biolreprod.103.017178)

Li M, Khambu B, Zhang H, Kang JH, Chen X, Chen D, Vollmer L, Liu PQ, Vogt A \& Yin XM 2013 Suppression of lysosome function induces autophagy via a feedback down-regulation of MTOR complex 1 (MTORC1) activity. Journal of Biological Chemistry 288 35769-35780. (https://doi.org/10.1074/jbc.M113.511212)

Li R, Zhou R, Wang H, Li W, Pan M, Yao X, Zhan W, Yang S, Xu L, Ding Y et al. 2019 Gut microbiota-stimulated cathepsin K secretion mediates TLR4-dependent M2 macrophage polarization and promotes tumor metastasis in colorectal cancer. Cell Death and Differentiation 26 2447-2463. (https://doi.org/10.1038/s41418-019-0312-y)

Liang S, Jiang H, Shen XH, Zhang JB \& Kim NH 2018 Inhibition of cathepsin $B$ activity prevents deterioration in the quality of in vitro aged porcine oocytes. Theriogenology 116 103-111. (https://doi.org/10.1016/j. theriogenology.2018.04.035)

Lindner GM \& Wright Jr RW 1983 Bovine embryo morphology and evaluation. Theriogenology 20 407-416. (https://doi.org/10.1016/0093691x(83)90201-7)

Liu L, Zhang N, Dou Y, Mao G, Bi C, Pang W, Liu X, Song D \& Deng H 2017 Lysosomal dysfunction and autophagy blockade contribute to IMB6G-induced apoptosis in pancreatic cancer cells. Scientific Reports 7 41862. (https://doi.org/10.1038/srep41862)

Lonergan P, Khatir H, Piumi F, Rieger D, Humblot P \& Boland MP 1999 Effect of time interval from insemination to first cleavage on the developmental characteristics, sex ratio and pregnancy rate after transfer of bovine embryos. Journal of Reproduction and Fertility 117 159-167. (https://doi.org/10.1530/jrf.0.1170159)

Marino G, Niso-Santano M, Baehrecke EH \& Kroemer G 2014 Selfconsumption: the interplay of autophagy and apoptosis. Nature Reviews: Molecular Cell Biology 15 81-94. (https://doi.org/10.1038/nrm3735)

Min SH, Song BS, Yeon JY, Kim JW, Bae JH, Park SY, Lee YH, Chang KT \& Koo DB 2014 A cathepsin B inhibitor, E-64, improves the preimplantation development of bovine somatic cell nuclear transfer embryos. Journal of Reproduction and Development 60 21-27. (https://doi.org/10.1262/ jrd.2013-074)

Mizushima N 2007 Autophagy: process and function. Genes and Development 21 2861-2873. (https://doi.org/10.1101/gad.1599207)

Mizushima N \& Levine B 2010 Autophagy in mammalian development and differentiation. Nature Cell Biology 12 823-830. (https://doi. org/10.1038/ncb0910-823)

Mizushima N, Yamamoto A, Matsui M, Yoshimori T \& Ohsumi Y 2004 In vivo analysis of autophagy in response to nutrient starvation using transgenic mice expressing a fluorescent autophagosome marker. Molecular Biology of the Cell 15 1101-1111. (https://doi.org/10.1091/ mbc.e03-09-0704)

Neuber E, Luetjens CM, Chan AW \& Schatten GP 2002 Analysis of DNA fragmentation of in vitro cultured bovine blastocysts using TUNEL. Theriogenology $57 \quad 2193-2202 . \quad$ (https://doi.org/10.1016/s0093691x(02)00901-9)

Pan Y, Wang M, Wang L, Xu G, Baloch AR, Kashif J, Fan J \& Yu S 2020 Interleukin-1 beta induces autophagy of mouse preimplantation embryos 
and improves blastocyst quality. Journal of Cellular Biochemistry 121 1087-1100. (https://doi.org/10.1002/jcb.29345)

Pezhman M, Hosseini SM, Ostadhosseini S, Rouhollahi Varnosfaderani S, Sefid F \& Nasr-Esfahani MH 2017 Cathepsin B inhibitor improves developmental competency and cryo-tolerance of in vitro ovine embryos. BMC Developmental Biology 17 10. (https://doi.org/10.1186/ s12861-017-0152-2)

Scaffidi C, Fulda S, Srinivasan A, Friesen C, Li F, Tomaselli KJ, Debatin KM, Krammer PH \& Peter ME 1998 Two CD95 (APO-1/Fas) signaling pathways. EMBO Journal 17 1675-1687. (https://doi.org/10.1093/ emboj/17.6.1675)

Shen X, Zhang N, Wang Z, Bai G, Zheng Z, Gu Y, Wu Y, Liu H, Zhou D \& Lei L 2015 Induction of autophagy improves embryo viability in cloned mouse embryos. Scientific Reports 5 17829. (https://doi.org/10.1038/ srep17829)

Simonsen A \& Tooze SA 2009 Coordination of membrane events during autophagy by multiple class III PI3-kinase complexes. Journal of Cell Biology 186 773-782. (https://doi.org/10.1083/jcb.200907014)

Singh T \& O'Reilly AM 2019 Autophagy as a beacon and sentry for aging stem cells. Autophagy 15 740-741. (https://doi.org/10.1080/15548627 .2019.1569948)

Song BS, Yoon SB, Kim JS, Sim BW, Kim YH, Cha JJ, Choi SA, Min HK, Lee Y, Huh JW et al. 2012 Induction of autophagy promotes preattachment development of bovine embryos by reducing endoplasmic reticulum stress. Biology of Reproduction 87 1-11. (https://doi.org/10.1095/ biolreprod.111.097949)

Song S, Tan J, Miao Y, Li M \& Zhang Q 2017 Crosstalk of autophagy and apoptosis: involvement of the dual role of autophagy under ER stress. Journal of Cellular Physiology 232 2977-2984. (https://doi.org/10.1002/ jсp.25785)

Thorburn A 2008 Apoptosis and autophagy: regulatory connections between two supposedly different processes. Apoptosis $131-9$. (https:// doi.org/10.1007/s10495-007-0154-9)

Tsukamoto S, Kuma A, Murakami M, Kishi C, Yamamoto A \& Mizushima N 2008 Autophagy is essential for preimplantation development of mouse embryos. Science 321 117-120. (https://doi.org/10.1126/ science.1154822)

Vancompernolle K, Van Herreweghe F, Pynaert G, Van De Craen M, De Vos K, Totty N, Sterling A, Fiers W, Vandenabeele P \& Grooten J 1998 Atractyloside-induced release of cathepsin B, a protease with caspase- processing activity. FEBS Letters 438 150-158. (https://doi.org/10.1016/ s0014-5793(98)01275-7)

Vandaele L, Mateusen B, Maes DG, De Kruif A \& Van Soom A 2007 Temporal detection of caspase-3 and -7 in bovine in vitro produced embryos of different developmental capacity. Reproduction 133 709-718. (https://doi.org/10.1530/REP-06-0109)

Vizovisek M, Fonovic M \& Turk B 2019 Cysteine cathepsins in extracellular matrix remodeling: extracellular matrix degradation and beyond. Matrix Biology 75-76 141-159. (https://doi.org/10.1016/j.matbio.2018.01.024)

Weidberg H, Shpilka T, Shvets E, Abada A, Shimron F \& Elazar Z 2011 LC3 and GATE- $16 \mathrm{~N}$ termini mediate membrane fusion processes required for autophagosome biogenesis. Developmental Cell 20 444-454. (https:// doi.org/10.1016/j.devcel.2011.02.006)

Werneburg NW, Guicciardi ME, Bronk SF \& Gores GJ 2002 Tumor necrosis factor-alpha-associated lysosomal permeabilization is cathepsin B dependent. American Journal of Physiology: Gastrointestinal and Liver Physiology 283 G947-G956. (https://doi.org/10.1152/ajpgi.00151.2002)

Wu D, Wang H, Li Z, Wang L, Zheng F, Jiang J, Gao Y, Zhong H, Huang Y \& Suo Z 2012 Cathepsin B may be a potential biomarker in cervical cancer. Histology and Histopathology 27 79-87. (https://doi.org/10.14670/HH27.79)

Yamanaka KI, Khatun H, Egashira J, Balboula AZ, Tatemoto H, Sakatani M, Takenouchi N, Wada Y \& Takahashi M 2018 Heat-shockinduced cathepsin B activity during IVF and culture compromises the developmental competence of bovine embryos. Theriogenology 114 293-300. (https://doi.org/10.1016/j.theriogenology.2018.04.005)

Yoshioka K, Suzuki C \& iwamura S 2000 Effects of activin A and follistatin on developmental kinetics of bovine embryos: cinematographic analysis in a chemically defined medium. Journal of Reproduction and Fertility 118 119-125. (https://doi.org/10.1530/jrf.0.1180119)

Received 23 January 2020

First decision 25 February 2020

Revised manuscript received 24 March 2020

Accepted 30 March 2020 